\title{
Gather Round: In Vitro Tumor Spheroids as Improved Models of In Vivo Tumors
}

\author{
Siddarth Chandrasekaran and Michael R. King* \\ Department of Biomedical Engineering, Cornell University, Ithaca, New York, USA
}

Continued efforts to understand cancer have progressed in parallel with tendencies of tumors to evade conventional treatments used to control tumor progression. Cancer cells fit the 'survival of the fittest' paradigm given the fact that they exist in an aberrant in vivo environment consisting of compromised blood supply, low oxygen concentration, large amounts of reducing equivalents, low $\mathrm{pH}$ and hypoxia to name a few [1]. This differs from the normal tissue environment, where a perfect homeostasis is maintained. These in vivo pathophysiological gradients play an important role in tumor progression and their response to treatment [2]. To illustrate this idea, we describe two of many examples where these gradients play an important role in determining the molecular basis of biological incongruities found in tumors. For instance, hypoxic conditions can make cells resistant to radio-ablative therapy because radiotherapy relies on DNA damage orchestrated by oxygen centered free radicals [3]. The gradient in glucose results in a gradient in cellular proliferation, resulting in a slower dividing cell population in the center of the tumor giving rise to cancer stem cells [4]. Thus, asking important questions about tumor progression and efficacy to drugs on cancer cells cultured as a conventional monolayer has little physiological relevance. Cancer cells cultured as 3D spheroids have brought cancer research a step closer to in vivo tumors by recreating the physiologically relevant gradients of factors such as nutrients, oxygen, $\mathrm{pH}$ and cellular proliferation (Figure 1). The past decade has seen several approaches for culturing cells as multicellular spheroids in which microfabrication and biomaterials have played important roles in facilitating new insights.

The most popular method for spheroid cell culture is the hanging drop technique where cells are grown in small droplets on an inverted surface [5]. However, the small media volume for so many cells in a $10-20 \mu \mathrm{L}$ droplet is a significant disadvantage of this method. Microfabrication has played an important role in developing 3D cell culture platforms for cancer research [6]. The advantages of microfabrication include high fidelity molding of micron-size surface topographies, chemical modification of the surface, and potential integration into microfluidic devices. Our group has developed a gas expansion molding (GEM) technique for generating microbubbles

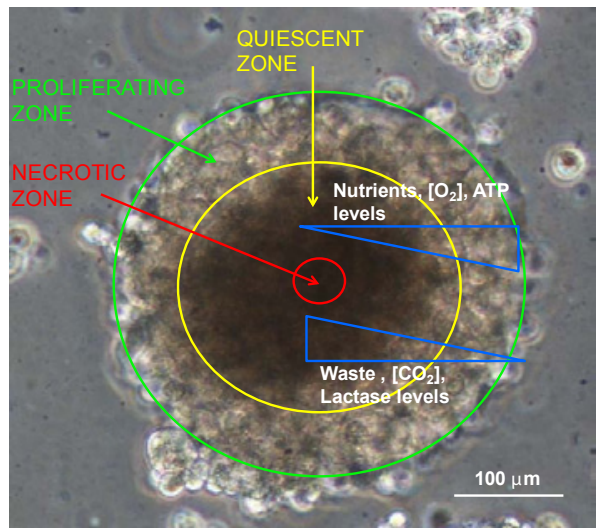

Figure 1: Pathophysiological gradients in a 3D tumor spheroid which are 80-150 $\mu \mathrm{m}$ diameter spherical compartments formed on polydimethylsiloxane (PDMS), a silicone based elastomeric polymer. We showed that arrays of spherical cavities formed on PDMS [7] using this technique can be used for culturing cancer cells as spheroids [8]. The unique geometry of microbubbles allows the cells to rapidly condition their microenvironment by secreting soluble factors to influence their function [9]. Also, perfusion culture system using microbubble arrays has shown that Colo205 cells cultured as spheroids under flow are more resistant to doxorubicin treatment resembling the actual disease condition [10].

Despite the obvious advantages of microfabricated cell culture platforms such as high-throughput design and easy integration with microfluidic systems, the size of spheroids that can be generated by these techniques is often limited by the geometry of the cell culture platform. The maximum number of cells that can be seeded onto microfabricated wells is often less than $10^{3}$. To mimic the primary tumor before vascularization the number of cells in a tumor spheroid should be more than a few thousand and size should be 400-600 $\mu \mathrm{m}$ in diameter [11], something that cannot be easily achieved by microfabricated cell culture platforms. An alternative approach to generate tumor spheroids to mimic a primary tumor is to culture them in rotating wall vessel bioreactors for an extended period of time up to 16 days [12]. While this method has been quite successful in generating spheroids of increased size, the time frame and equipment cost remain limiting factors. The use of biocompatible polymers for spheroid cell culture has been po pular since 1970s. In fact, the very first method for propagating cancer cells as multicellular aggregates involved culturing them on agar [13]. Hydrophobic polymers hinder cells from adhering to the substrate, resulting in increased cell-cell interaction and enabling their propagation as spheroids. We showed that WM115, a metastatic melanoma cell line cultured on PDMS showed altered spheroid morphology with increased presence of cells expressing stem-cell markers when compared to cells propagating as a monolayer [14]. Breast cancer cells propagating as 3D spheroids on PDMS showed increased expression of E-selectin ligands and had a significantly stronger interaction with human recombinant E-selectin when compared to monolayer cells [15]. This supports the idea that studying important aspects of tumor progression makes a significant difference when cancer cell lines are cultured as tumor spheroids.

While monoculture of tumor spheroids have been of importance,

${ }^{*}$ Corresponding author: Michael R. King, Department of Biomedical Engineering, Cornell University, Ithaca, New York, USA, E-mail: mike.king@cornell.edu

Received October 22, 2012; Accepted October 23, 2012; Published October 25, 2012

Citation: Chandrasekaran S, King MR (2012) Gather Round: In Vitro Tumor Spheroids as Improved Models of In Vivo Tumors. J Bioengineer \& Biomedical Sci 2:e109. doi:10.4172/2155-9538.1000e109

Copyright: (C) 2012 Chandrasekaran S, et al. This is an open-access article distributed under the terms of the Creative Commons Attribution License, which permits unrestricted use, distribution, and reproduction in any medium, provided the original author and source are credited. 
Citation: Chandrasekaran S, King MR (2012) Gather Round: In Vitro Tumor Spheroids as Improved Models of In Vivo Tumors. J Bioengineer \& Biomedical Sci 2:e109. doi:10.4172/2155-9538.1000e109

Page 2 of 2

spheroid co-culture is a tool that is gaining increased attention in cancer research. Tumors in vivo are vastly heterogeneous, consisting of cancer cells with different levels of aggressiveness with infiltrating endothelial cells, cancer associated fibroblasts and macrophages [16]. Heterogeneity results from heterotypic interactions that are believed to play a role in determining the ability of a cell to metastasize from the primary site. This heterogeneity could be partly captured in vitro by co-culturing cell lines with different metastatic potential. Co-culturing non-tumorigenic mammary epithelial cell line MCF10A, with the weakly metastatic MCF7 cell line and the highly metastatic BT20 cell line as 3D spheroids resulted in favoring the E-selectin mediated adhesion of the highly metastatic BT20 cell line [15]. The co-culture conditions also increased the invasiveness of the most aggressive cell subpopulation. Thus, co-culture tumor spheroids can closely mimic the in vivo homotypic and heterotypic interactions offering a platform for addressing important questions about cancer metastasis in physiologically relevant scenarios.

In summary, spheroid monoculture and co-culture could potentially replace conventional monolayer culture in cancer research. Though cancer cell lines offer several advantages such as ease in handling, unlimited passages in vitro and considerable heterogeneity; all of these advantages risk being lost when cultured as a 2D monolayer. The advent of microfabrication and biomaterials have paved the way for $3 \mathrm{D}$ culture of cancer cells which offers all these advantages in an in vivo-like environment recreating a cellular microenvironment with cell-cell homotypic and heterotypic interactions.

\section{Acknowledgment}

The work described was supported by the Cornell Center on the Microenvironment \& Metastasis through Award Number U54CA143876 from the National Cancer Institute. The content is solely the responsibility of the authors and does not represent the official views of the National Cancer Institute or the National Institutes of Health.

\section{References}

1. Vaupel P, Kallinowski F, Okunieff $P$ (1989) Blood flow, oxygen and nutrien supply, and metabolic microenvironment of human tumors: a review. Cancer Res 49: 6449-6465.

2. Hirschhaeuser F, Menne H, Dittfeld C, West J, Mueller-Klieser W, et al. (2010)
Multicellular tumor spheroids: an underestimated tool is catching up again. $J$ Biotechnol 148: 3-15

3. Moeller B, Richardson R, Dewhirst M (2007) Hypoxia and radiotherapy: opportunities for improved outcomes in cancer treatment. Cancer and Metastasis Reviews. 26: 241-248.

4. Moore N, Lyle S (2011) Quiescent, Slow-Cycling Stem Cell Populations in Cancer: A Review of the Evidence and Discussion of Significance. Journal of Oncology.

5. Timmins NE, Nielsen LK (2007) Generation of multicellular tumor spheroids by the hanging-drop method. Methods Mol Med 140: 141-151.

6. Griffith LG, Swartz MA (2006) Capturing complex 3D tissue physiology in vitro. Nat Rev Mol Cell Biol 7: 211-224.

7. Giang UB, Lee D, King MR, DeLouise LA (2007) Microfabrication of cavities in polydimethylsiloxane using DRIE silicon molds. Lab Chip 7: 1660-1662.

8. Giang UBT, King MR, DeLouise LA (2008) Microfabrication of Bubbular Cavities in PDMS for Cell Sorting and Microcell Culture Applications. J Bionic Eng. 5 308-316.

9. Chandrasekaran S, Giang UB, King MR, DeLouise LA (2011) Microenvironment induced spheroid to sheeting transition of immortalized human keratinocytes ( $\mathrm{HaCaT})$ cultured in microbubbles formed in polydimethylsiloxane. Biomaterials 32: 7159-7168.

10. Agastin S, Giang UB, Geng Y, DeLouise LA, King MR (2011) Continuously perfused microbubble array for 3D tumor spheroid model. Biomicrofluidics. 5 : 24110

11. Nyga A, Cheema U, Loizidou M (2011) 3D tumour models: novel in vitro approaches to cancer studies. J Cell Commun Signal 5: 239-248.

12. Ong SM, Zhao Z, Arooz T, Zhao D, Zhang S, et al. (2010) Engineering scaffold-free 3D tumor model for in vitro drug penetration studies. Biomaterials 31: $1180-1190$.

13. Hamburger AW, Salmon SE (1977) Primary bioassay of human tumor stem cells. Science. 197: 461-463.

14. Chandrasekaran S, DeLouise LA (2011) Enriching and characterizing cance stem cell sub-populations in the WM115 melanoma cell line. Biomaterials 32 9316-9327.

15. Chandrasekaran S, Geng Y, Delouise LA, King MR (2012) Effect of homotypic and heterotypic interaction in 3D on the E-selectin mediated adhesive properties of breast cancer cell lines. Biomaterials 33: 9037-9048.

16. Hanahan D, Weinberg RA (2011) Hallmarks of cancer: the next generation Cell 144: 646-674. 